\title{
Estrutura populacional dos camarões simpátricos Potimirim glabra e Potimirim potimirim (Crustacea, Decapoda, Atyidae) no rio Sahy, Rio de Janeiro, Brasil
}

\author{
Giovana V. Lima, Charlene M. Silveira \& Lídia M. Y. Oshiro
}

Estação de Biologia Marinha, Universidade Federal Rural do Rio de Janeiro, Rua Sereder s/nº Itacuruçá, Mangaratiba, 23880-000 Rio de Janeiro, Brasil. (giovalverde@yahoo.com.br; oshiro@ufrrj.br)

\begin{abstract}
Populational structure of the sympatric freshwater shrimps Potimirim glabra and Potimirim potimirim (Crustacea, Decapoda, Atyidae) in the Sahy River, Rio de Janeiro, Brazil. The aim of this work was to get some knowledge about the populational structure of the two sympatric freshwater shrimp species, Potimirim glabra (Kingsley, 1878) and Potimirim potimirim (Müller, 1881) in the Sahy River, Mangaratiba, Rio de Janeiro, Brazil. The specimens were sampled monthly from September 1997 to February 1999 by sieving the marginal vegetation, on rocky surface and among litter on river bottom, with 15-minute effort per sampling period. The animals were sorted sexed and their total and cephalothoracic length were measured. A total of 4,889 individuals were collected in Sahy river: 3,281 P. glabra and 1,608 P. potimirim. The sex ratio for all collected individuals was 1:1 for P. glabra and 1:2.3 for P. potimirim. In both populations, five sexual maturity stages were determined and the populations were constituted mainly of adult freshwater shrimps. This study shows the difference between the recruitment of juveniles along these species. The recruitment for P. glabra occurs throughout the year, except in Spring, while P. potimirim was recorded only in Autumn. The seasonal distribution of ovigerous females of P. glabra is similar to P. potimirim, with reproduction on Spring and Summer.
\end{abstract}

KEYWORDS. Atyidae, freshwater shrimps, populational structure, Potimirim, recruitment.

RESUMO. Este trabalho teve por objetivo obter conhecimento sobre a estrutura populacional de dois camarões de água doce simpátricos, Potimirim glabra (Kingsley, 1878) e Potimirim potimirim (Müller, 1881) no rio Sahy, Mangaratiba, Rio de Janeiro, Brasil. Os indivíduos foram coletados mensalmente durante o período de setembro de 1997 a fevereiro de 1999 utilizando-se peneiras, que foram passadas sob a vegetação marginal, superfície de rochas e pequenas poças d'água, num esforço de 15 minutos por coletor. Os animais foram separados quanto ao sexo e mensurados em relação ao comprimento total e do cefalotórax. Um total de 4.889 indivíduos foram coletados no rio Sahy: 3.281 P. glabra e 1.608 P. potimirim. A razão sexual observada foi de 1:1 para $P$. glabra e $1: 2,3$ para $P$. potimirim. Em ambas populações, cinco estágios de maturidade sexual foram determinados, sendo as populações constituídas principalmente por camarões adultos. O recrutamento de juvenis apresentou diferenças em ambas as espécies. O recrutamento de $P$. glabra ocorreu durante todo o período de estudo, exceto na primavera, enquanto que $P$. potimirim foi registrado somente no outono. A distribuição sazonal de fêmeas ovígeras de P. glabra é similar ao de P. potimirim, com reprodução na primavera e no verão.

PALAVRAS-CHAVE. Atyidae, camarões de água doce, estrutura populacional, Potimirim, recrutamento.

A família Atyidae representa um importante componente dos ecossistemas subtropicais e tropicais de água doce, estando atualmente constituída por mais de 20 gêneros (Holthuis, 1955). Esses animais exercem papel fundamental na cadeia trófica dos ambientes límnicos (BENZIE, 1982) e no processo de renovação do sedimento (Moulton et al. 2004; Souza \& Moulton, 2005).

As espécies descritas de Potimirim Holthuis, 1954 encontram-se distribuídas nas Américas do Norte, Central e do Sul (Barros \& Fontoura, 1996b); o gênero está composto por cinco espécies: P. americana (GuérinMéneville, 1855), P. mexicana De Saussure, 1857, P. glabra (Kingsley, 1878), P. potimirim (Müller, 1881) e P. brasiliana Villalobos, 1959. No Brasil, são registradas apenas $P$. glabra, nos Estados do Rio de Janeiro, São Paulo e Santa Catarina, e $P$. potimirim, nos Estados de Pernambuco, Alagoas, Bahia, Rio de Janeiro, São Paulo e Santa Catarina (Paim et al., 1997; Ramos-Porto \& Coelho, 1998; Lima \& Oshiro, 1999 e Melo, 2003). Villalobos (1959) revisou Potimirim e descreveu $P$. brasiliana, uma nova espécie para o território brasileiro; todavia, SMALley (1963) contrapôs a revisão, incluindo a espécie como sinonímia de P. glabra.

A estrutura populacional representa uma importante ferramenta no estudo de diferentes grupos, pois permite conhecer a amplitude de tamanho alcançado pelos indivíduos de uma população, suas freqüências e flutuações ao longo dos diferentes tamanhos, o que representa uma fonte de taxas contínuas de mortalidade e natalidade (Díaz \& Conde, 1989; Hartnoll \& BRyant, 1990 e BEGON et al., 1996).

Apesar da importância ecológica atribuída à família, poucos foram os estudos realizados com o grupo, verificando-se, tanto para $P$. glabra quanto para $P$. potimirim, pesquisas restritas à reprodução, crescimento e ecologia (BARROs \& FontOURA, 1996a, b; LiMA \& OSHIRO, 1999, 2002a, b; MARTÍNEZ-MAYÉn \& ROMÁN-CONTRERAS, 2003; Moulton et al., 2004 e Souza \& Moulton, 2005).

Objetiva-se obter informações sobre a estrutura populacional de duas espécies simpátricas de camarões de água doce no rio Sahy, Potimirim glabra e $P$. potimirim, verificando-se sua distribuição em relação ao tamanho, freqüência sazonal, razão sexual e recrutamento de juvenis. 


\section{MATERIAL E MÉTODOS}

Os exemplares foram obtidos mensalmente de setembro de 1997 a fevereiro de 1999 ao longo do rio Sahy $\left(22^{\circ} 56^{\prime} \mathrm{S} ; 4^{\circ} 01^{\prime} \mathrm{W}\right)$, Município de Mangaratiba, Estado do Rio de Janeiro, Brasil, em seis diferentes áreas de amostragem (Fig. 1). As áreas foram separadas segundo distâncias em relação à jusante, declividade do terreno, tipo de rocha e presença de vegetação marginal. As estações I e II foram os pontos mais distantes em relação à foz, localizados a $2 \mathrm{~km}$ da foz, apresentando elevada declividade do terreno, com trechos encachoeirados, correnteza das águas acentuada, inúmeras rochas de grande porte e vegetação marginal composta principalmente por árvores. As estações III e IV foram os pontos intermediários, localizados a $1,5 \mathrm{~km}$ da foz, com declividade média, correnteza reduzida, algumas rochas de pequeno porte e seixos, com uma vegetação marginal densa, composta por pequenos arbustos e gramíneas. As estações V e VI, obtidas na jusante, apresentam influência constante da salinidade, declividade reduzida do terreno, com ausência total de trechos encachoeirados, correnteza muito reduzida, presença de areia, seixos, e vegetação abundante, constituída principalmente por Spartina alterniflora Loisel, 1890 (Poaceae).

Durante o estudo, a salinidade foi verificada utilizando-se um refratômetro.

Os animais foram coletados com auxílio de peneiras com $50 \mathrm{~cm}$ de diâmetro e $2 \mathrm{~mm}$ de abertura de malha que foram passadas simultaneamente ao longo das margens do rio, sob a vegetação marginal e pequenas poças, num esforço de 15 minutos por dois coletores.

Todos os exemplares obtidos foram colocados em sacos plásticos identificados e armazenados em caixa térmica com gelo, para evitar perda de apêndices e danos entre os indivíduos em processo de muda.

No laboratório, as amostras foram mantidas congeladas no freezer até o processamento. Após descongelamento, em temperatura ambiente, os exemplares foram identificados, triados por sexo, contados e medidos. Todos os indivíduos foram mensurados em relação ao comprimento total (medida compreendida entre a órbita e a extremidade distal do telso), e o comprimento do cefalotórax (medida compreendida entre a órbita e a extremidade posterior do cefalotórax), utilizando-se um paquímetro de $0,1 \mathrm{~mm}$ de precisão. Os exemplares foram distribuídos em 14 classes de tamanho, com comprimento total entre 1,1 e 26,0 mm.

Os animais foram separados em cinco estágios de maturidade sexual, de acordo com a morfologia do apêndice masculino (Tab. I), enquanto as fêmeas e juvenis foram identificados segundo a maturidade sexual populacional observada por Lima \& OshiRo (1999, 2002a).

O Teste t de Student $(\alpha=0,05)$ foi empregado para comparação do tamanho observado entre machos e fêmeas de ambas populações. A razão sexual foi comparada através do Teste do qui-quadrado $\left(x^{2}\right)$ para verificar a proporção obtida entre machos e fêmeas ao longo dos meses de coleta, enquanto o recrutamento foi observado relacionando-se a proporção de juvenis e o total de camarões capturados mensalmente.

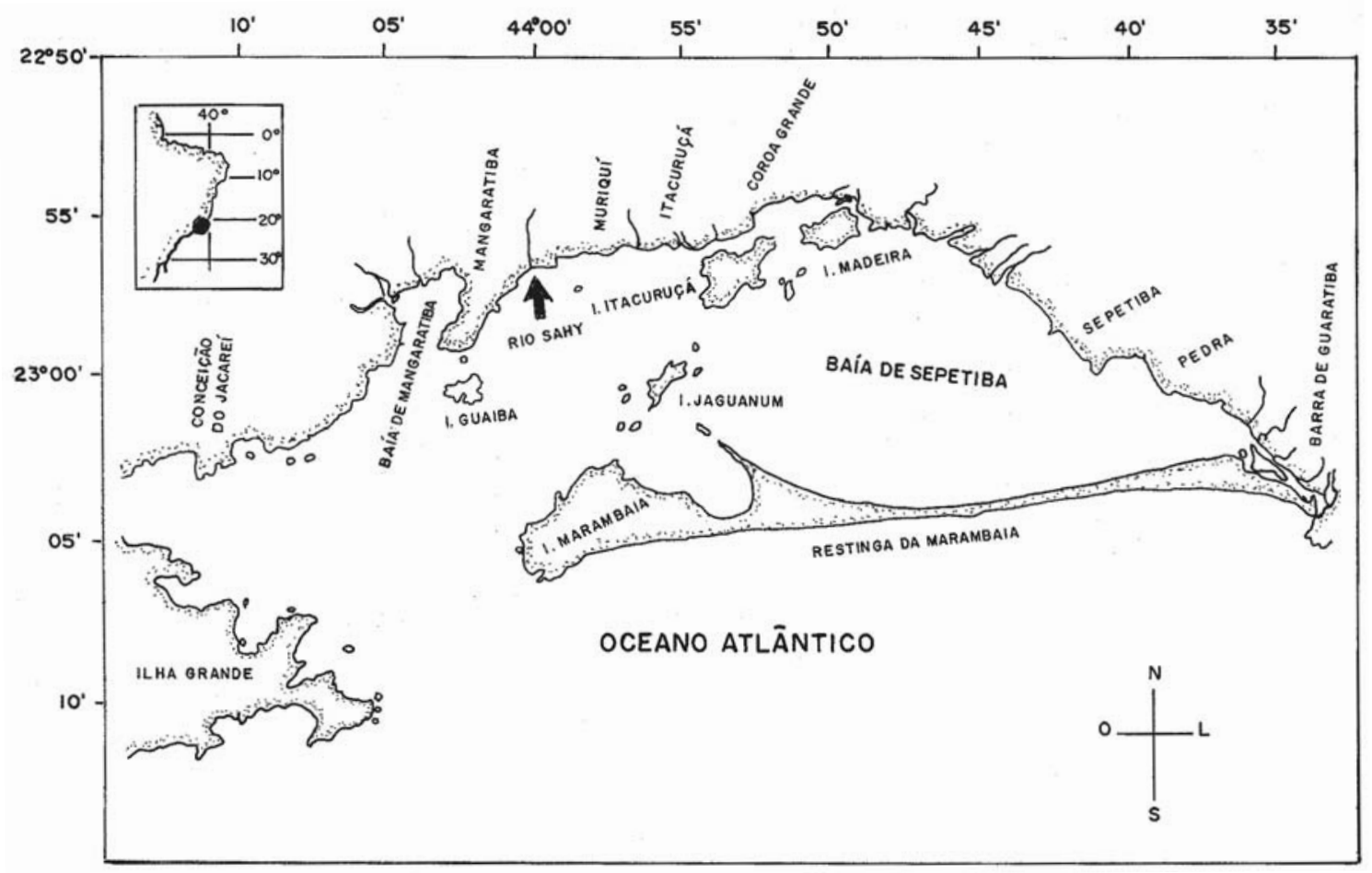

Fig. 1. Mapa da área de estudo na Baía de Sepetiba, Rio de Janeiro, Brasil. 
Tabela I. Estágios de maturidade sexual observados para Potimirim glabra (Kingsley, 1878) e P. potimirim (Müller, 1881), rio Sahy, Rio de Janeiro, durante o período de setembro de 1997 a fevereiro de 1999.

\begin{tabular}{|c|c|c|c|}
\hline $\begin{array}{l}\text { Estágios de maturidade } \\
\text { sexual }\end{array}$ & Características morfológicas & $\begin{array}{c}\text { P. glabra } \\
\text { Comprimento total }(\mathrm{mm})\end{array}$ & $\begin{array}{c}\text { P. potimirim } \\
\text { Comprimento total }(\mathrm{mm})\end{array}$ \\
\hline Juvenis & Ausência de caracteres sexuais secundários & $<8,0$ & $<6,5$ \\
\hline Machos imaturos & $\begin{array}{l}\text { Apêndice masculino incompleto ou } \\
\text { em desenvolvimento }\end{array}$ & $8,0-11,0$ & $6,5-9,5$ \\
\hline Machos maturos & $\begin{array}{l}\text { Apêndice masculino completo ou } \\
\text { desenvolvido }\end{array}$ & $>11,0$ & $>9,5$ \\
\hline Fêmeas imaturas & $\begin{array}{l}\text { Ausência do apêndice masculino e de ovos } \\
\text { aderidos aos pleópodos }\end{array}$ & $8,0-16,0$ & $6,5-9,9$ \\
\hline Fêmeas maturas & $\begin{array}{l}\text { Ausência do apêndice masculino e presença } \\
\text { ou ausência de ovos aderidos aos pleópodos }\end{array}$ & $>16,0$ & $>10,0$ \\
\hline
\end{tabular}

\section{RESULTADOS E DISCUSSÃO}

Foram capturados 4.889 camarões ao longo do período de coleta, sendo 3.281 exemplares de $P$. glabra e 1.608 de P. potimirim. Barros \& Fontoura (1996b) capturaram 9.318 indivíduos de P. glabra no Estado de Santa Catarina, o que corresponde a três vezes a quantidade obtida neste estudo. Provavelmente, o menor número de animais coletados no rio Sahy decorre de uma população menor, distribuída num ambiente com elevada densidade de vegetação e substrato ao longo do rio, o que permite reduzida exposição e vulnerabilidade dos espécimes; ou ainda, decorrente de diferenças durante o esforço de coleta, seja pelo número de estações, tempo ou número de coletores.

De acordo com Chace \& Hobbs (1969) e Ramos-Porto \& PalÁcios (1981), esses atídeos encontram-se distribuídos ao longo dos rios sempre sob a vegetação marginal, em diferentes microhábitats escolhidos segundo a presença de seixos, areia e correnteza. Estão sempre ocupando os mesmos ambientes naturais, porém em freqüências antagônicas, demonstrando preferências diferenciadas e partição ambiental, possivelmente por possuírem a mesma valência trófica (LimA \& Oshiro, 2002b).

A razão sexual observada para a população de $P$. glabra não diferiu de 1:1, enquanto para a população de $P$. potimirim verificou-se uma proporção estatisticamente diferente da esperada, apresentando uma razão de 1:2,3. A razão sexual observada para $P$. glabra apresentou predominância de machos durante a primavera e verão, verificando-se maior número de fềmeas durante o outono $\mathrm{e}$ inverno, de abril a setembro de 1998. Por outro lado, $P$. potimirim apresentou elevado número de machos em todas as estações do ano, exceto nos meses de julho e agosto de 1998, durante o inverno (Tab. II, Fig. 2).

As diferenças observadas em relação à razão sexual podem ser explicadas através dos diferentes tamanhos e mortalidade observados entre machos e fêmeas (WILSON \& PianKa, 1963). Segundo Botelho et al. (2001), fatores como a muda, dispersão e reprodução também podem explicar essas diferenças na proporção sexual. No entanto, essas diferenças também podem estar relacionadas a problemas amostrais. De acordo com Martínez-Mayén \& RomÁn-Contreras (2003) e Barros \& Fontoura (1996a), P. glabra apresenta tanto no rio Coyuca, México quanto na praia da Vigia, Brasil, uma proporção sexual de 1:1, assim como no presente estudo. Potimirim glabra e P. potimirim apresentaram semelhanças em relação à distribuição das freqüências ao longo dos diferentes estágios de maturidade sexual. Os machos maturos de ambas as populações ocorreram em maiores percentagens, enquanto os juvenis e os machos imaturos foram observados em reduzida freqüência (Fig. 3). Essa diferença na freqüência entre os indivíduos

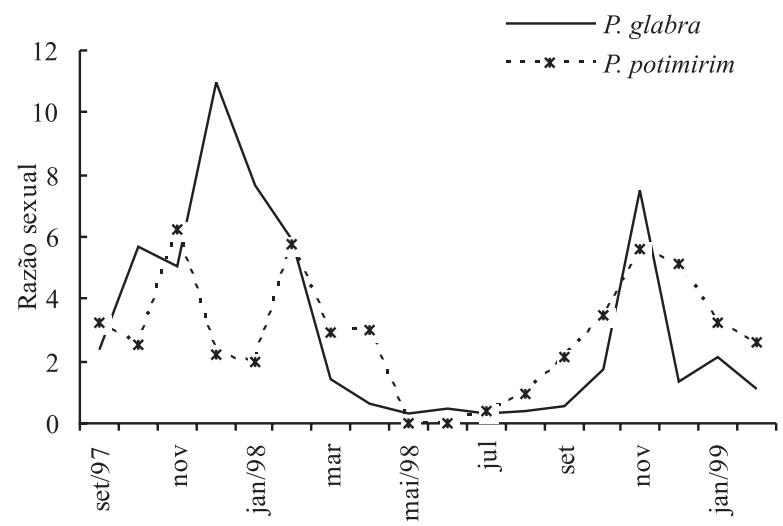

Fig. 2. Razão sexual mensal de Potimirim glabra (Kingsley, 1878) e $P$. potimirim (Müller, 1881), capturados no rio Sahy, Rio de Janeiro, durante o período de setembro de 1997 a fevereiro de 1999.

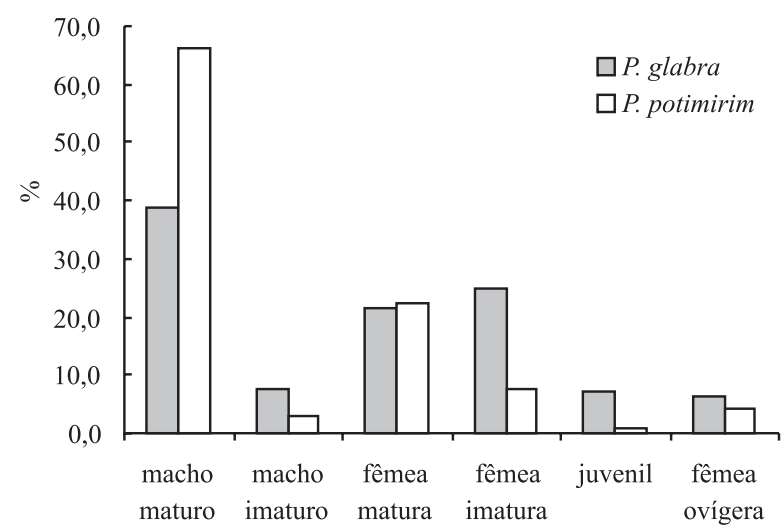

Fig. 3. Distribuição dos diferentes estágios de maturidade sexual de Potimirim glabra (Kingsley, 1878) e P. potimirim (Müller, 1881), capturados no rio Sahy, Rio de Janeiro, durante o período de setembro de 1997 a fevereiro de 1999. 
Tabela II. Razão sexual de Potimirim glabra (Kingsley, 1878) e P. potimirim (Müller, 1881), rio Sahy, Rio de Janeiro, durante o período de setembro de 1997 a fevereiro de 1999 (* Diferença significativa ao nível de significância de 5\%).

\begin{tabular}{|c|c|c|c|c|c|c|}
\hline \multirow[b]{2}{*}{ Meses/ano } & \multicolumn{3}{|c|}{ Potimirim glabra } & \multicolumn{3}{|c|}{ Potimirim potimirim } \\
\hline & $\begin{array}{c}\mathrm{N}^{\circ} \\
\text { Machos }\end{array}$ & $\begin{array}{c}\mathrm{N}^{\circ} \\
\text { Fêmeas }\end{array}$ & Razão sexual & $\begin{array}{c}\mathrm{N}^{\circ} \\
\text { Machos }\end{array}$ & $\begin{array}{c}\mathrm{N}^{\circ} \\
\text { Fêmeas }\end{array}$ & Razão sexual \\
\hline Setembro/1997 & 66 & 28 & $1: 2,36 *$ & 13 & 4 & $1: 3,25^{*}$ \\
\hline Outubro/1997 & 34 & 6 & $1: 5,66^{*}$ & 25 & 10 & $1: 2,50 *$ \\
\hline Novembro/1997 & 86 & 17 & $1: 5,06^{*}$ & 94 & 15 & $1: 6,26^{*}$ \\
\hline Dezembro/1997 & 55 & 5 & $1: 11,00 *$ & 24 & 11 & $1: 2,18^{*}$ \\
\hline Janeiro/1998 & 61 & 8 & $1: 7,62 *$ & 66 & 33 & $1: 2,00^{*}$ \\
\hline Fevereiro/1998 & 65 & 11 & $1: 5,91 *$ & 58 & 10 & $1: 5,80^{*}$ \\
\hline Março/1998 & 70 & 49 & $1: 1,43$ & 93 & 32 & $1: 2,91^{*}$ \\
\hline Abril/1998 & 100 & 160 & $1: 0,62 *$ & 12 & 4 & $1: 3,00^{*}$ \\
\hline Maio/1998 & 26 & 88 & $1: 0,29 *$ & - & - & - \\
\hline Junho/1998 & 85 & 192 & $1: 0,44^{*}$ & - & - & - \\
\hline Julho/1998 & 50 & 153 & $1: 0,33^{*}$ & 44 & 117 & $1: 0,37^{*}$ \\
\hline Agosto/1998 & 83 & 200 & $1: 0,41^{*}$ & 73 & 79 & $1: 0,92$ \\
\hline Setembro $19 / 98$ & 160 & 288 & $1: 0,56^{*}$ & 132 & 62 & $1: 2,12 *$ \\
\hline Outubro/1998 & 136 & 80 & $1: 1,70 *$ & 70 & 20 & $1: 3,50^{*}$ \\
\hline Novembro/1998 & 150 & 20 & $1: 7,50^{*}$ & 259 & 46 & $1: 5,63^{*}$ \\
\hline Dezembro/1998 & 120 & 91 & $1: 1,32 *$ & 62 & 12 & $1: 5,16^{*}$ \\
\hline Janeiro/1999 & 100 & 46 & $1: 2,17^{*}$ & 48 & 15 & $1: 3,20^{*}$ \\
\hline Fevereiro/1999 & 83 & 75 & 1: 1,11 & 39 & 15 & $1: 2,60^{*}$ \\
\hline Total & 1.530 & 1.517 & 1: 1,01 & 1.112 & 485 & $1: 2,29^{*}$ \\
\hline
\end{tabular}

coletados pode estar associada a um erro na amostragem (Hartnoll \& Bryant, 1990) ou talvez devido a uma diferença na dispersão dos indivíduos ao longo da área de coleta. De acordo com Lima \& Oshiro (2002b), os espécimes apresentam ampla seleção de hábitats ao longo do rio; os indivíduos maturos de $P$. glabra estão associados a um ambiente de declive, com rochas e correnteza, enquanto os de $P$. potimirim estão associados a águas de menor correnteza, sem rochas, com margens ricas em vegetação e leito com elevada densidade de folhiço em decomposição. Este fato pode ter facilitado a captura dos espécimes, uma vez que eram facilmente visualizados sobre este substrato. Já os juvenis de ambas as espécies, segundo estes autores, necessitam de maiores refúgios ao longo da vegetação marginal, de declividade reduzida, ambiente lêntico e raso, o que permite águas mais aquecidas e conseqüentemente elevando a ação do processo de muda e crescimento.

Os indivíduos de ambas espécies apresentaram uma variação similar em relação ao tamanho. Potimirim glabra variou entre 4,1 e 26,0 mm de comprimento total, sendo as fêmeas com 8,1 a 26,0 mm e os machos 8,1 e 22,0 mm. Potimirim potimirim, todavia, registrou tamanho reduzido, variando entre 2,1 e $26,0 \mathrm{~mm}$, com fêmeas entre 6,1 e 26,0 mm e machos entre 6,1 e 20,0 mm. A distribuição em classes de tamanho apresentou distribuição unimodal entre os machos e bimodal entre as fêmeas (Fig. 4).

A distribuição unimodal dos dados observada neste estudo é um padrão comum entre os crustáceos tropicais, sendo resultado de uma desova contínua, recrutamento, migração e mortalidade (DíAz \& CONDE, 1989), enquanto que a bimodalidade indica sazonalidade nos eventos reprodutivos e de recrutamento (SEIPLE, 1979; SpIVAK et al., 1991; Tsuchida \& WatANABE, 1997).

O dimorfismo sexual observado ocorre em relação ao apêndice masculino (Tab. I) e devido ao maior tamanho apresentado pelas fêmeas. Segundo GHERARDi \& Micheli (1989), entre os crustáceos é comum que os machos atinjam maiores dimensões, provavelmente para
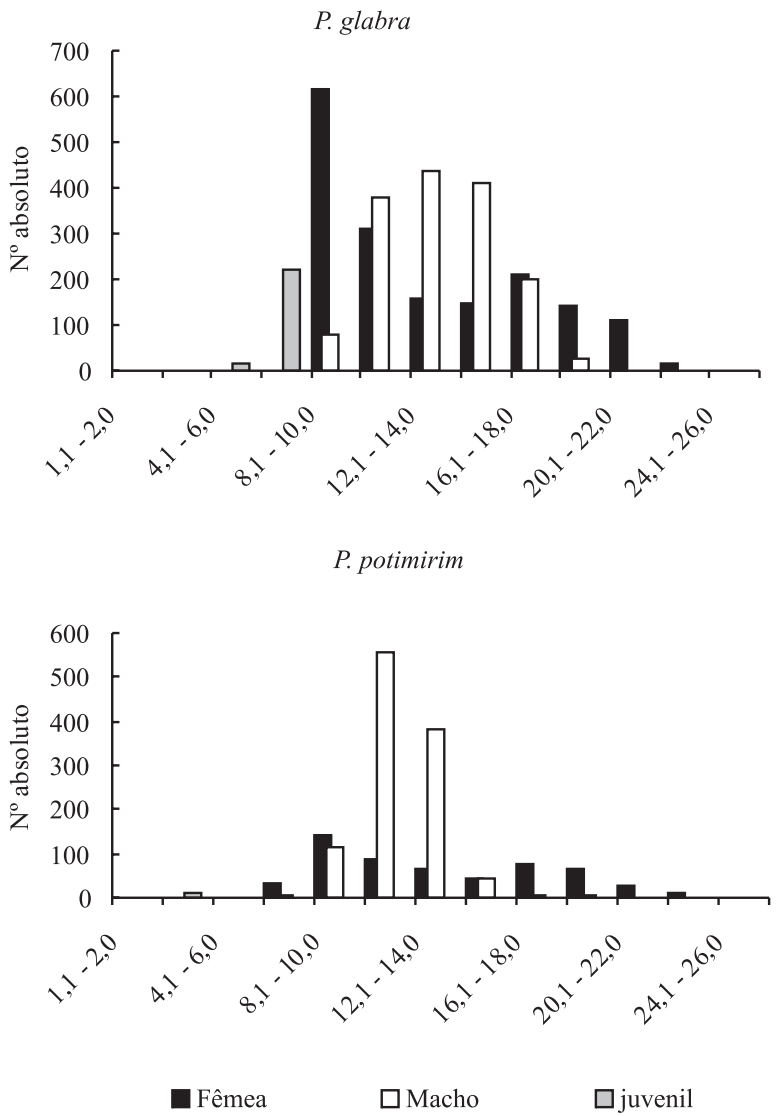

Fig. 4. Distribuição de machos, fêmeas e juvenis de Potimirim glabra (Kingsley, 1878) e P. potimirim (Müller, 1881) por classes de tamanho, capturados no rio Sahy, Rio de Janeiro, durante o período de setembro de 1997 a fevereiro de 1999. 
assegurar a cópula e garantir a competição intraespecífica. No entanto, o maior crescimento das fêmeas verificado neste estudo pode maximizar o potencial reprodutivo da espécie através de uma maior fecundidade (PARKer, 1992), enquanto que o pequeno tamanho observado entre os machos pode ser compensado pela elevada densidade junto às fêmeas (BARROs \& FonTOURA, 1996b). ABrams (1988) menciona que a energia dispensada ao crescimento de machos é direcionada ao metabolismo estrutural do indivíduo, e não à produção de ovos, garantindo assim habilidades na fertilização e nas lutas intraespecíficas. De acordo com SANTOS et al. (2001), em estudos com camarões comerciais, a estratégia de maior tamanho entre as fêmeas também pode ser constatada em Litopenaeus schmitti (Burkenroad, 1936),
Farfantepenaeus subtilis (Pérez-Farfante, 1967) e Xiphopenaeus kroyeri (Heller, 1862).

Os juvenis ocorreram somente entre as classes de 4,1 a 8,0 mm para $P$. glabra e entre 2,1 a 4,0 $\mathrm{mm}$ para $P$. potimirim (Fig. 4). O recrutamento de juvenis na população de $P$. glabra foi verificado durante todo o estudo, exceto na primavera; para $P$. potimirim ocorreu durante o outono (Fig. 5). Provavelmente, este processo foi registrado diferentemente em ambas as populações, em decorrência de uma distribuição espacial diferenciada ou devido à competição interespecífica ao longo do rio (Lima \& Oshiro, 2002b). Segundo Santos (1978), a reprodução pode interferir em relação à entrada de novos indivíduos na população, através do tipo e freqüência de desova, fecundidade e comportamento reprodutivo.

\section{P. glabra}

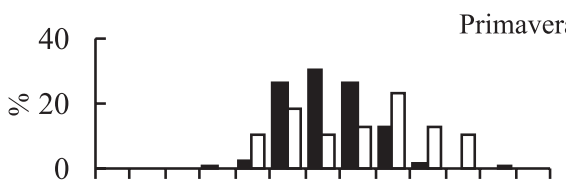

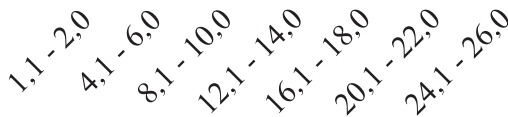
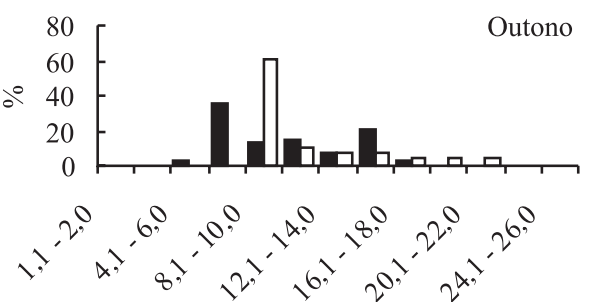
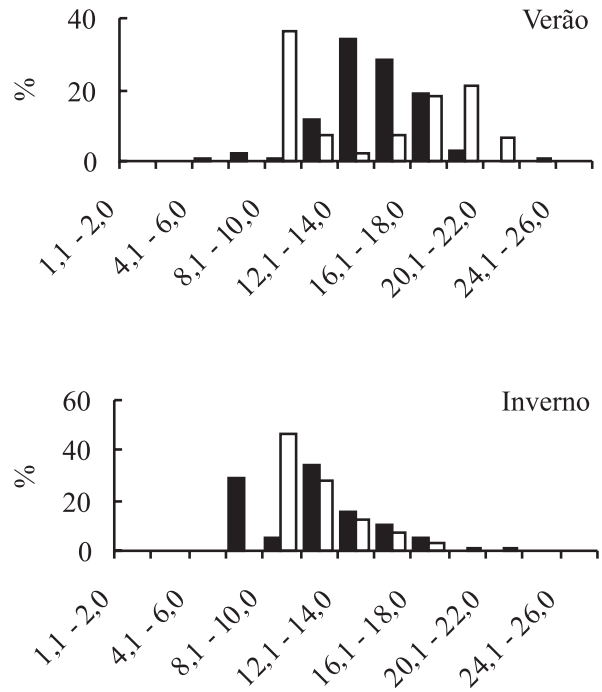

P. potimirim
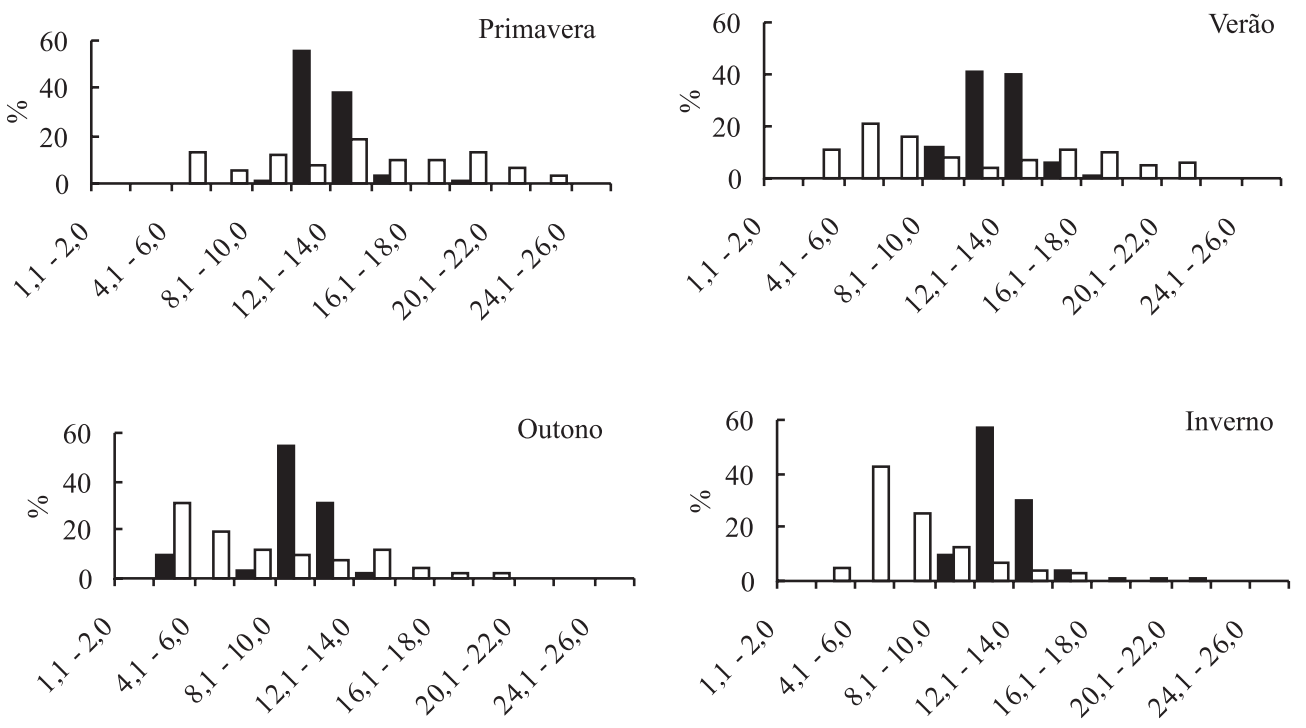

$\square$ Macho

Fêmea

Fig. 5. Proporção sexo-etária de Potimirim glabra (Kingsley, 1878) e P. potimirim (Müller, 1881), em diferentes estações do ano no rio Sahy, Rio de Janeiro, durante o período de setembro de 1997 a fevereiro de 1999. 
Os juvenis de $P$. glabra foram observados em maior abundância durante o outono (19\%), resultante da desova de verão. No verão, todavia, a freqüência de juvenis ficou em torno de $2 \%$, embora tenha ocorrido uma elevada freqüência de fêmeas ovígeras durante a primavera e o verão. Provavelmente a reduzida presença de juvenis durante estas estações pode ser explicada pela ação da temperatura no desenvolvimento e maturação dos tecidos gonadais (ADIYODY \& ADIYODY, 1970; WEAR, 1974) e sua ação catalítica sobre as diferentes fases larvais (Boolootian et al., 1959). Segundo EMmerson (1994), uma estratégia reprodutiva comum entre os crustáceos consiste em realizar a postura durante o verão, quando as temperaturas estão elevadas e o fitoplâncton mais abundante, o que facilita o desenvolvimento dos novos indivíduos.

Os juvenis de $P$. potimirim foram coletados somente no outono, possivelmente devido a um crescimento mais rápido durante os meses mais quentes, isto é, durante a primavera e verão. Já no inverno, provavelmente nenhum exemplar foi amostrado devido à vulnerabilidade da espécie a baixas temperaturas (Gore et al., 1978) ou decorrente da necessidade de microhábitats compostos por substratos de diferentes tamanhos granulométricos. Todavia, BARros \& Fontoura (1996b) não atribuíram a ausência de juvenis durante o mês de fevereiro às reduzidas temperaturas, mas à seca do riacho, acompanhada de dificuldades para a captura dos indivíduos.

PINHEIRO et al. (1994) relatam que a temperatura pode mudar a taxa de desenvolvimento nos crustáceos, promovendo a redução ou a aceleração das formas embrionárias ou pós-embrionárias. Segundo CAVALCANTE et al. (1986), o camarão Macrobrachium rosenbergii (De Man, 1879) adota migrações para áreas de menor profundidade onde emergem a porção dorsal, a fim de minimizar a influência de temperaturas reduzidas, menores que $15^{\circ} \mathrm{C}$. Este fato parece totalmente condizente para as espécies de Atyidae observadas, já que indivíduos menores foram capturados em locais de pouca profundidade.

Fêmeas ovígeras de P. glabra e P. potimirim foram encontradas em maiores percentagens durante a primavera, verão e outono, corroborando as informações de Barros \& Fontoura (1996a), Lima \& Oshiro (1999; 2002a). Por outro lado, as fêmeas que não se reproduziram durante o outono saíram dos nichos mais protegidos, provavelmente para realizarem uma nova muda e conseqüentemente, nova cópula, em pontos com maiores temperaturas, o que permitiu maior suscetibilidade desses indivíduos à captura.

Agradecimentos. Aos Drs. Marcelo A. A. Pinheiro (UNESP, São Vicente, SP) e Lenício Gonçalves (UFRRJ), pelos comentários e valiosas sugestões fornecidas à dissertação apresentada ao Curso de Ciências Ambientais e Florestais da UFRRJ, e ao funcionário Casemiro A. Alves pelo auxílio nas coletas mensais.

\section{REFERÊNCIAS BIBLIOGRÁFICAS}

Abrams, P. A. 1988. Sexual difference in resource use in hermit crabs: consequences and causes. In: Chelazzi, G. \& VANnini, M. eds. Behavioral adaptation to intertidal life. Plenum,
New York. p.283-296

Adiyodi, K. G. \& Adiyodi, R. G. 1970. Endocrine control of reproduction in decapoda crustacea. Biological Review 45:121-165.

Barros, M. P. \& Fontoura, N. F. 1996a. Biologia reprodutiva de Potimirim glabra (Kingsley, 1878) (Crustacea, Decapoda, Atyidae), na Praia da Vigia, Garopaba, Santa Catarina, Brasil. Nauplius $4: 1-10$.

1996b. Crescimento de Potimirim glabra (Kingsley, 1878) (Crustacea, Decapoda, Atyidae), na Praia da Vigia, Garopaba, Santa Catarina, Brasil. Nauplius 4:11-28.

Begon, M.; Mortimer, M. \& Thompson, D. J. 1996. Population ecology. A unified study of animals and plants. $3^{\mathrm{a}} \mathrm{ed}$. London, Blackwell Science. 204p.

Benzie, J. A. H. 1982. The complete larval development of Caridina mcculochi Roux, 1929 (Decapoda, Atyidae) reared in the laboratory. Journal of Crustacean Biology 2(4):493513.

Boolootian, R. A. A.; Giese, A. C.; Farmanfarmain, A. \& Tucker, J. 1959. Reproductive cycles of five west coast crabs. Physiological Zoology 32:213-220.

Botelho, E. R. O.; Santos, M. C. F. \& Souza, J. R. B. 2001. Aspectos populacionais do Guaiamum, Cardisoma guanhumi Latreille, 1825, do estuário do Rio Una (Pernambuco - Brasil). Boletim Técnico Científico, CEPENE 9(1):123-146.

Cavalcante, L. B.; Correa, E. S. \& Cordeiro, E. A. 1986. Camarão. Manual de cultivo do Macrobrachium rosenbergii (pitu havaiano - gigante da Malásia). Aquaculture 21:375-385.

Chace, F. A. \& Hobbs, H. H., JR. 1969. The freshwater and terrestrial Decapoda crustaceans of the West Indies special reference to Dominica. Bulletin of the United States National Museun 292:1-243.

Díaz, H. \& Conde, J. E. 1989. Population dynamics and life history of the mangrove crab Aratus pisonii (Brachyura, Grapsidae) in a marine environment. Bulletin of Marine Science 45(1):148-163.

EMmerson, W. D. 1994. Seasonal breeding cycles and sex ratio of eight species of crabs from Magazana, a mangrove estuary in Transkei, Southern Africa. Journal of Crustacean Biology 14(3):568-578.

Gherardi, F. \& Micheli, F. 1989. Relative growth and population structure of the freshwater crab, Potamon potamios palestinensis, in the dead sea area (Israel). Israel Journal of Zoology 36:133-145.

Gore, K.; Kulczycki, G. R. \& Hastings, P. A. 1978. A second ocurrence of the Brazilian freshwater shrimp, Potimirim potimirim, along the Central Eastern Florida Coast. Florida Scientist 41(1):57-61.

Hartnoll, R. G. \& Bryant, A. D. 1990. Size-frequency distributions in Decapod Crustacea - The quick, the dead, and the castoffs. Journal of Crustacean Biology 10(1):14-19.

Holthuis, L. B. 1955. The recent genera of the Caridean and Stenopodidean shrimps (Class Crustacea, order Decapoda, supersection Natantia) with keys for their determination. Zoologische Verhandelingen 26:1-157.

Lima, G. V. \& Oshiro, L. M. Y. 1999. Aspectos reprodutivos do camarão de água doce Potimirim potimirim (Müller, 1881) (Crustacea, Decapoda, Atyidae) do Rio Sahy, Mangaratiba/RJ. Nauplius 7:65-71.

2002a. Aspectos reprodutivos de Potimirim glabra (Kingsley,1878) (Crustacea, Decapoda, Atyidae) no Rio Sahy, Mangaratiba, RJ. Revista Brasileira de Zoologia 19(3):861866.

. 2002b. Partição ambiental de Potimirim glabra (Kingsley) e Potimirim potimirim (Muller) (Crustacea, Decapoda, Atyidae) no rio Sahy, Mangaratiba, Rio de Janeiro, Brasil. Revista Brasileira de Zoologia 19(supl.2):175-179.

Martínez-Mayén, M. \& Román-Contreras, R. 2003. Reproducción de Potimirim glabra (Kingsley, 1878) (Crustacea: Decapoda: Atyidae) en el río Coyuca, Guerrero, México. Contribuciones al Estudio de los Crustáceos del Pacífico Este 2:103115 .

Melo, G. A. S. 2003. Famílias Atyidae, Palemonidae e Sergestidae. In: Melo, G. A. S. ed. Manual de identificação dos Crustacea Decapoda de água doce do Brasil. São Paulo, Loyola. p.289-415. 
Moulton, T. P.; Souza, M. L.; Silveira, R. M. L. \& Krsulovic, F. A M. 2004. Effects of ephemeropterans and shrimps on periphyton and sediments in a coastal stream (Atlantic Forest, Rio de Janeiro, Brazil). Journal of the North American Benthological Society 23(4):868-881.

Paim, J. P.; Peso-Aguiar, M. C.; Carqueja, C. R. G.; Almeida, T. C. A. \& Assis, R. C. F. 1997. Ocorrência de Potimirim potimirim (Müller, 1881) (Crustacea, Decapoda, Atyidae) no Rio MucuriBahia. Nauplius 5(2):147-148.

Parker, G. A. 1992. The evolution of sexual dimorphism in fish. Journal of Fish Biology 41(b): 1-20.

Pinheiro, M. A. A.; Fransozo, A. \& Negreiros-Fransozo, M. L. 1994. Estimativa da duração larval em função da temperatura para a família Majidae (Crustacea, Decapoda, Brachyura). Boletim do Instituto de Pesca 21:75-81.

Ramos-Porto, M. \& Coelho, P. A. 1998. Malacostraca. Eucarida (Alpheoidea excluded). In: Young, P. S. ed. Catalogue of Crustacea of Brazil. Museu Nacional, Rio de Janeiro. p.325-350.

Ramos-Porto, M. \& Palácios, J. A. P. 1981. Estudos ecológicos do rio Capibaribe-Mirim, PE. IV. Crustáceos decápodas natantes. Trabalhos de Oceanografia da Universidade Federal de Pernambuco 16:265-296.

Santos, E. P. 1978. Dinâmica de populações biológicas aplicada à pesca e piscicultura. São Paulo, EDUSP. $129 \mathrm{p}$.

Santos, M. C. F.; Botelho, E. R. O. \& Ivo, C. T. C. 2001. Biologia populacional e manejo da pesca de aratu, Goniopsis cruentata (Latreille, 1803) (Crustacea: Decapoda: Grapsidae) no litoral sul de Pernambuco - Brasil. Boletim
Técnico Científico, CEPENE 9(1):87-123.

SEIPLE, W. 1979. Distribution, habitat preferences and breeding periods in the crustaceans Sesarma cinereum and $S$. reticulatum (Brachyura: Decapoda: Grapsidae). Marine Biology 52:7786.

Smalley, A. E. 1963. The genus Potimirim in Central America (Crustacea, Atyidae). Revista de Biologia Tropical 11(2): 177-183.

SouzA, M. L. \& Moulton, T. P. 2005. The effects of shrimps on benthic material in a Brazilian island stream. Freshwater Biology 50:592-602.

Spivak, E. D.; Gavio, M. A. \& Navarro, C. E. 1991. Life history and structure of the World's Southernmost Uca population: Uca uruguayensis (Crustacea, Brachyura) in Mar Chiquita lagoon (Argentina). Bulletin of Marine Science 48(3):679688.

Tsuchida, S. \& Watanabe, S. 1997. Growth and reproduction of the grapsid crab Plagusia dentipes (Decapoda: Brachyura). Journal of Crustacean Biology 17(1):90-97.

Villalobos, A. F. 1959. Contribución al conocimiento de los Atyidae de México. II (Crustacea, Decapoda). Estudio de algunas especies del genero Potimirim (=Ortmannia), com descripcion de una espécie nueva en Brasil. Anales del Instituto de Biología 30:269-330.

Wear, R. G. 1974. Incubation in British decapod Crustacea, and the effects of the temperature on the rate and success of embryonic development. Journal of the Marine Biological Association of the United Kington 54:745-762.

Wilson, M. F. \& PiankA, E. R. 1963. Sexual selection, sex ratio and mating system. American Naturalist 97:405-407.

Recebido em abril de 2005. Aceito em dezembro de 2005. ISSN 0073-4721

Artigo disponível em: www.scielo.br/isz 